\title{
Exact Robust Stability Analysis of Uncertain Systems with a Scalar Parameter via LMIs
}

\author{
Graziano Chesi \\ Department of Electrical and Electronic Engineering \\ The University of Hong Kong \\ Contact: http://www.eee.hku.hk/ ${ }^{\sim}$ chesi
}

\begin{abstract}
This paper provides an exact linear matrix inequality (LMI) condition for robust asymptotic stability of uncertain systems depending polynomially on a scalar parameter in both continuous-time and discrete-time cases. Specifically, this condition exploits sum of squares (SOS) techniques and is based on the construction of polynomials of known degree that detect the presence of eigenvalues on the boundary of the stability region. It is shown that this condition requires a much smaller computational burden than existing exact LMI conditions which might be prohibitive even for small scale systems.
\end{abstract}

\section{Introduction}

It is well-known that establishing whether an uncertain system is robustly asymptotically stable (i.e., asymptotically stable for all admissible uncertainties) is a key problem in automatic control. In the literature, several methods have been proposed in the case of linear systems affected by timeinvariant uncertainty. The dependence of the system on the uncertainty is typically linear or polynomial, while the uncertainty is typically represented by a vector constrained into a polytope.

Most of these methods provide in general sufficient conditions for robust asymptotic stability based on LMIs, see e.g. some of the pioneering works $[1,2]$. In some cases such as $[3,4,5,6,7]$ the conservatism can be reduced by increasing the degree of some polynomials used to build the LMIs, for instance representing a Lyapunov function candidate or a multiplier. Interestingly, this sometimes allows complete elimination of the conservatism, 
which means that these conditions are not only sufficient but also necessary. See [8] and references therein for details.

Unfortunately, the necessity of such conditions is generally based on unknown degrees of the polynomials used. As a consequence, one only knows that there exist sufficiently large degrees such that such conditions are also necessary, however nothing is generally known in about these degrees. For the case where the uncertainty is a scalar parameter, an exact LMI condition has been proposed in [9] for the continuous-time case by providing a bound on the degree of the Lyapunov function, however its computational burden rapidly increases with the system sizes as discussed in Sections 3-4.

This paper provides an exact LMI condition for establishing robust asymptotic stability of uncertain systems depending polynomially on a scalar parameter constrained in both continuous-time and discrete-time cases. Specifically, this condition exploits SOS techniques and is based on the construction of polynomials of known degree that detect the presence of eigenvalues on the boundary of the stability region. It is shown that this condition requires a much smaller computational burden than existing exact LMI conditions which might be prohibitive even for small scale systems.

\section{Problem Formulation}

Notation: $\mathbb{R}, \mathbb{C}$ : real and complex number sets; $I$ : identity matrix (of size specified by the context); 0: null matrix (of size specified by the context); $A \geq 0$ : symmetric positive definite matrix; $A^{\prime}$ : transpose of $A$; $\operatorname{det}(A)$ : determinant of $A ; \operatorname{spec}(A)=\{\lambda \in \mathbb{C}: \operatorname{det}(\lambda I-A)=0\} ; \mathrm{CT}, \mathrm{DT}$ : continuous-time and discrete-time.

Let us consider the uncertain system

$$
\left\{\begin{array}{l}
(\mathrm{CT} \text { case }) \dot{x}(t)=A(p) x(t) \\
(\mathrm{DT} \text { case }) x(t+1)=A(p) x(t) \\
p \in\left[p_{\min }, p_{\max }\right]
\end{array}\right.
$$

where $t \in \mathbb{R}$ is the time, $x(t) \in \mathbb{R}^{n}$ is the state, and $p$ is the uncertain parameter with bounds $p_{\min }, p_{\max } \in \mathbb{R}$. The function $A: \mathbb{R} \rightarrow \mathbb{R}^{n \times n}$ is polynomial of degree less than or equal to $d$, i.e.

$$
A(p)=\sum_{i=0}^{d} p^{i} A_{i}
$$

for some matrices $A_{0}, A_{1}, \ldots, A_{d} \in \mathbb{R}^{n \times n}$. 
The matrix $A(p)$ is said asymptotically stable in the CT case if $A(p)$ is Hurwitz (i.e., with all eigenvalues having negative real part) and in the DT case if it is Schur (i.e., with all eigenvalues having magnitude smaller than one). We consider the following problem.

Problem: to establish whether $A(p)$ is robustly asymptotically stable, i.e. $A(p)$ is asymptotically stable for all $p \in[0,1]$.

\section{Proposed Results}

With $G \in \mathbb{R}^{n \times n}$, let us define $\Phi(G) \in \mathbb{R}^{(n-1) \times(n-1)}$ as

$$
\Phi(G)=\left(\begin{array}{cccc}
g_{n-1} & g_{n-3} & g_{n-5} & \cdots \\
1 & g_{n-2} & g_{n-4} & \cdots \\
0 & g_{n-1} & g_{n-3} & \cdots \\
0 & 1 & g_{n-2} & \cdots \\
\vdots & \vdots & \vdots & \ddots
\end{array}\right)
$$

where $g_{0}, \ldots, g_{n-1} \in \mathbb{R}$ are the coefficients of the characteristic polynomial of $G$ according to

$$
\operatorname{det}(\lambda I-G)=\lambda^{n}+\sum_{j=0}^{n-1} g_{j} \lambda^{j}
$$

Also, with $G \in \mathbb{R}^{n \times n}$, let us denote with $\Psi(G) \in \mathbb{R}^{n(n-1) / 2 \times n(n-1) / 2}$ the matrix whose entries are the determinants of the $2 \times 2$ submatrices of $G$ according to

$$
(\Psi(G))_{j, k}=\operatorname{det}\left(\begin{array}{ll}
G_{y_{1}, z_{1}} & G_{y_{1}, z_{2}} \\
G_{y_{2}, z_{1}} & G_{y_{2}, z_{2}}
\end{array}\right)
$$

where $\left(y_{1}, z_{1}\right)$ and $\left(y_{2}, z_{2}\right)$ are the $j$-th and $k$-th pairs in the sequence $(1,2)$, $(1,3), \ldots,(1, n),(2,3),(2,4), \ldots,(2, n),(3,4), \ldots,(n-1, n)$.

Next, let us define

$$
\begin{aligned}
& \text { (CT case) } \quad i_{\max }=2 \\
& \text { (DT case) } \quad i_{\max }=3 .
\end{aligned}
$$

For all $i=1, \ldots, i_{\text {max }}$, let us introduce $f_{i}(p)$ as

$$
(\mathrm{CT} \text { case })\left\{\begin{array}{l}
f_{1}(p)=\operatorname{det}(-A(p)) \\
f_{2}(p)=\operatorname{det}(\Phi(A(p)))
\end{array}\right.
$$


or

$$
\text { (DT case) }\left\{\begin{array}{l}
f_{1}(p)=\operatorname{det}(I-A(p)) \\
f_{2}(p)=\operatorname{det}(I+A(p)) \\
f_{3}(p)=\operatorname{det}(I-\Psi(A(p))) .
\end{array}\right.
$$

Each function $f_{i}(p)$ is a polynomial of degree $\delta_{i}$ satisfying

$$
(\mathrm{CT} \text { case })\left\{\begin{array}{l}
\delta_{1} \leq d n \\
\delta_{2} \leq d n(n-1) / 2
\end{array}\right.
$$

or

$$
\text { (DT case) }\left\{\begin{array}{l}
\delta_{i} \leq d n \forall i=1,2 \\
\delta_{3} \leq d n(n-1)
\end{array}\right.
$$

Let us define

$$
\begin{aligned}
b\left(p, m_{i}\right) & =\left(1, p, \ldots, p^{m_{i}}\right)^{\prime} \\
m_{i} & =\left\lceil\delta_{i} / 2\right\rceil .
\end{aligned}
$$

Let $F_{i}=F_{i}^{\prime} \in \mathbb{R}^{\left(m_{i}+1\right) \times\left(m_{i}+1\right)}$ be a matrix such that

$$
f_{i}(p)=b\left(p, m_{i}\right)^{\prime} F_{i} b\left(p, m_{i}\right) .
$$

Such a matrix can be defined as

$$
\left(F_{i}\right)_{j, k}= \begin{cases}f_{i, j+k-2} & \text { if } j=k \\ f_{i, j+k-2} / 2 & \text { if }|j-k|=1 \\ 0 & \text { otherwise }\end{cases}
$$

where $f_{i, 0}, \ldots, f_{i, 2 m_{i}} \in \mathbb{R}$ are the coefficients of $f_{i}(p)$ according to

$$
f_{i}(p)=\sum_{j=0}^{2 m_{i}} f_{i, j} p^{j}
$$

Let $R_{i}=R_{i}^{\prime} \in \mathbb{R}^{\left(m_{i}+1\right) \times\left(m_{i}+1\right)}$ be a matrix such that

$$
1=b\left(p, m_{i}\right)^{\prime} R_{i} b\left(p, m_{i}\right) .
$$

Such a matrix can be defined as

$$
\left(R_{i}\right)_{j, k}= \begin{cases}1 & \text { if } j=k=1 \\ 0 & \text { otherwise }\end{cases}
$$

With $\Gamma_{i}=\Gamma_{i}^{\prime} \in \mathbb{R}^{m_{i} \times m_{i}}$, let $S_{i}\left(\Gamma_{i}\right)=S_{i}\left(\Gamma_{i}\right)^{\prime} \in \mathbb{R}^{\left(m_{i}+1\right) \times\left(m_{i}+1\right)}$ be a matrix such that

$$
\left(p-p_{\min }\right)\left(p_{\max }-p\right) \gamma_{i}(p)=b\left(p, m_{i}\right)^{\prime} S_{i}\left(\Gamma_{i}\right) b\left(p, m_{i}\right)
$$


where

$$
\gamma_{i}(p)=b\left(p, m_{i}-1\right)^{\prime} \Gamma_{i} b\left(p, m_{i}-1\right) .
$$

Such a matrix can be defined as

$$
\begin{aligned}
S_{i}\left(\Gamma_{i}\right)= & \frac{p_{\min }+p_{\max }}{2}\left(\left(\begin{array}{cc}
0_{m_{i}} & \Gamma_{i} \\
0 & 0_{m_{i}}^{\prime}
\end{array}\right)+\left(\begin{array}{cc}
0_{m_{i}}^{\prime} & 0 \\
\Gamma_{i} & 0_{m_{i}}
\end{array}\right)\right) \\
& -\left(\begin{array}{cc}
0 & 0_{m_{i}}^{\prime} \\
0_{m_{i}} & \Gamma_{i}
\end{array}\right)-p_{\min } p_{\max }\left(\begin{array}{cc}
\Gamma_{i} & 0_{m_{i}} \\
0_{m_{i}}^{\prime} & 0
\end{array}\right) .
\end{aligned}
$$

Lastly, let $T_{i}\left(\Delta_{i}\right)=T_{i}\left(\Delta_{i}\right)^{\prime} \in \mathbb{R}^{\left(m_{i}+1\right) \times\left(m_{i}+1\right)}$ be a linear parametrization of the linear subspace

$$
\mathcal{T}_{i}=\left\{T=T^{\prime}: b\left(p, m_{i}\right)^{\prime} T b\left(p, m_{i}\right)=0 \forall p \in \mathbb{R}\right\}
$$

where $\Delta_{i} \in \mathbb{R}^{c_{i}}$ is a free vector and $c_{i}$ is the dimension of $\mathcal{T}_{i}$ given by [10]

$$
c_{i}=m_{i}\left(m_{i}-1\right) / 2
$$

Theorem 1 The matrix $A(p)$ is robustly asymptotically stable if and only if the following conditions hold:

- $A\left(p_{0}\right)$ is asymptotically stable, where $p_{0}$ is an arbitrary number in $\left[p_{\min }, p_{\max }\right]$;

- for all $i=1, \ldots, i_{\max }$, there exist $\beta_{i} \in \mathbb{R}, \Gamma_{i}=\Gamma_{i}^{\prime} \in \mathbb{R}^{m_{i} \times m_{i}}$ and $\Delta_{i} \in \mathbb{R}^{c_{i}}$ satisfying the LMIs

$$
\left\{\begin{aligned}
\beta_{i} & >0 \\
\Gamma_{i} & \geq 0 \\
F_{i}-\beta_{i} R_{i}-S_{i}\left(\Gamma_{i}\right)+T_{i}\left(\Delta_{i}\right) & \geq 0 .
\end{aligned}\right.
$$

Proof. " $\Leftarrow$ " (Sufficiency) Suppose that $A\left(p_{0}\right)$ is asymptotically stable and for all $i=1, \ldots, i_{\max }(22)$ holds. Let us define

$$
w_{i}(p)=b\left(p, m_{i}\right)^{\prime}\left(F_{i}-\beta_{i} R_{i}-S_{i}\left(\Gamma_{i}\right)+T_{i}\left(\Delta_{i}\right)\right) b\left(p, m_{i}\right)
$$

and let us observe that $\gamma_{i}(p)$ and $w_{i}(p)$ are nonnegative due to the second and third LMIs in (22). From (12), (15), (17) and (20) it follows that

$$
\begin{aligned}
0 & \leq w_{i}(p) \\
& =f_{i}(p)-\beta_{i}-\left(p-p_{\min }\right)\left(p_{\max }-p\right) \gamma_{i}(p) .
\end{aligned}
$$


Since $\beta_{i}>0$ from the first LMI in (22), one has that

$$
f_{i}(p)>0 \quad \forall p \in\left[p_{\min }, p_{\max }\right] .
$$

In the CT case, this implies that no eigenvalue of $A(p)$ lies on the imaginary axis for $p \in\left[p_{\min }, p_{\max }\right]$ since $f_{1}(p)=\operatorname{det}(-A(p))$ and $f_{2}(p)$ (which is the $(n-1)$-Hurwitz determinant of $A(p))$ satisfies [8]

$$
f_{2}(p)=\prod_{i=1, \ldots, n-1 ; j=i+1, \ldots, n}-\left(\lambda_{i}(A(p))+\lambda_{j}(A(p))\right)
$$

where $\lambda_{1}(A(p)), \ldots, \lambda_{n}(A(p))$ are the eigenvalues of $A(p)$. In the DT case, this implies that no eigenvalue of $A(p)$ lies on the unit circumference for $p \in\left[p_{\min }, p_{\max }\right]$ since $f_{1}(p)=\operatorname{det}(I-A(p)), f_{2}(p)=\operatorname{det}(I+A(p))$ and $[8]$

$$
f_{3}(p)=\prod_{i=1, \ldots, n-1 ; j=i+1, \ldots, n} \lambda_{i}(A(p)) \lambda_{j}(A(p)) .
$$

Since the eigenvalues of $A(p)$ are continuous with respect to $p$ and that $A\left(p_{0}\right)$ is asymptotically stable, it follows that $A(p)$ is asymptotically stable for $p \in\left[p_{\min }, p_{\max }\right]$.

" $\Rightarrow$ " (Necessity) Suppose that $A(p)$ is robustly asymptotically stable. This implies that $A\left(p_{0}\right)$ is asymptotically stable. Moreover, since the eigenvalues of $A(p)$ are continuous with respect to $p$, it follows that no eigenvalue of $A(p)$ lies on the imaginary axis in the CT case (or on the unitary circumference in the DT case) for all $p \in\left[p_{\min }, p_{\max }\right]$. Since $A\left(p_{0}\right)$ is asymptotically stable, it follows that

$$
0<f_{i}(p) \forall p \in\left[p_{\min }, p_{\max }\right] \forall i=1, \ldots, i_{\max } .
$$

Since $\left[p_{\min }, p_{\max }\right]$ is bounded and $f_{i}(p)$ is polynomial, this is equivalent to the existence of $\beta_{i}>0$ such that

$$
0 \leq f_{i}(p)-\beta_{i} \quad \forall p \in\left[p_{\min }, p_{\max }\right] \forall i=1, \ldots, i_{\max }
$$

Since $p$ is a scalar, this is equivalent to the existence of a SOS polynomial $\gamma_{i}(p)$ such that $w_{i}(p)$ is SOS, see e.g. [10]. From the same reference one has that a polynomial in one variable is SOS if and only if it has a positive semidefinite Gram matrix. The proof is concluded observing that $\Gamma_{i}$ is a generic Gram matrix of $\gamma_{i}(p)$ and that $F_{i}-\beta_{i} R_{i}-S_{i}\left(\Gamma_{i}\right)+T_{i}\left(\Delta_{i}\right)$ is a generic Gram matrix of $w_{i}(p)$. In fact, these Gram matrices are hence positive semidefinite, and the LMIs (22) consequently hold. 


\begin{tabular}{c|ccccc}
$\eta^{W C}$ & $d=1$ & 2 & 3 & 4 & 5 \\
\hline$n=2$ & 4 & 7 & 15 & 22 & 36 \\
3 & 10 & 20 & 52 & 74 & 130 \\
4 & 15 & 54 & 119 & 210 & 327 \\
5 & 36 & 127 & 291 & 502 & 796 \\
$\eta_{Z T I}^{W C}$ & $d=1$ & 2 & 3 & 4 & 5 \\
\hline$n=2$ & 22 & 76 & 162 & 280 & 430 \\
3 & 174 & 501 & 1179 & 1884 & 3066 \\
4 & 666 & 2116 & 4894 & 8104 & 12994 \\
5 & 1810 & 6820 & 15030 & 26440 & 41050
\end{tabular}

Table 1: Worst-case total number of LMI scalar variables for Theorem 1 in the $\mathrm{CT}$ case (i.e., $\eta^{W C}$ ) and for Corollary 5.2 in [9] (i.e., $\eta_{Z T I}^{W C}$ ).

Theorem 1 provides a sufficient and necessary condition for establishing whether $A(p)$ is robustly asymptotically stable via the LMIs (22) for $i=$ $1, \ldots, i_{\max }$, which can be considered either simultaneously or separately. The total number of LMI scalar variables is given by

$$
\eta=\sum_{i=1}^{i_{\max }}\left(1+m_{i}\left(m_{i}+1\right) / 2+c_{i}\right) .
$$

In the worst-case, which occurs when all the inequalities in (9)-(10) are equations (and this happens when the matrix $A_{d}$ has full rank), one has

$$
\begin{array}{ll}
(\text { CTcase }) & \eta^{W C}=2+\lceil d n / 2\rceil^{2}+\lceil d n(n-1) / 4\rceil^{2} \\
(\text { DTcase }) & \eta^{W C}=3+2\lceil d n / 2\rceil^{2}+\lceil d n(n-1) / 2\rceil^{2}
\end{array}
$$

It is interesting to observe that this number is significantly smaller than that of existing exact LMI conditions. In particular, Corollary 5.2 in [9] provides an exact LMI condition for the CT case whose worst-case total number of LMI scalar variables is

$$
\eta_{Z T I}^{W C}=\left(n(n+1) m_{p}+n^{2}\left(\left\lceil m_{p} / 2\right\rceil+d\right)^{2}\right) / 2
$$

where $m_{p}=d(n(n+1) / 2-1)$ (the worst-case for this condition occurs, e.g., when at least one of the matrices $A_{1}, \ldots, A_{d}$ has full rank). Table 1 shows $\eta^{W C}$ for the CT case and $\eta_{Z T I}^{W C}$ for some values of $n$ and $d$. 


\section{Examples}

Let us consider (1) in the CT case with

$$
A(p)=\left(\begin{array}{cccc}
0 & 1 & 0 & 2-p \\
-1-p^{2} & -2 & 7 p-1 & 0 \\
-p^{3} & 1-p & -1 & 0 \\
p & 0 & p^{4} & -1
\end{array}\right)
$$

and $\left[p_{\min }, p_{\max }\right]=[0,1]$. Hence, $n=4$ and $d=4$. Let us use Theorem 1 . We have that $A(0)$ is asymptotically stable, but the LMIs (22) cannot be fulfilled for $i=2$ (the maximum $\beta$ fulfilling (22) for $i=2$ is -0.808 ). Hence, from Theorem 1 we conclude that $A(p)$ is not robustly asymptotically stable. Indeed, this is true since $A(0.638)$ has a pair of eigenvalues with real part equal to 0.008 .

It is interesting to observe that the same conclusion might not be obtained using existing LMI conditions due to the huge computational burden required. Indeed, for the exact LMI condition provided by Corollary 5.2 in [9], the total number of LMI scalar variables in this example is indeed given by Table 1 and is equal to $\eta_{Z T I}^{W C}=8104$ : our Matlab crashes attempting to solve this LMI problem (standard PC with Windows 7, SeDuMi toolbox, Intel Core Quad $3 \mathrm{GHz}$, 4GB RAM).

On the other hand, the computational burden required by the proposed approach is significantly smaller: an upper bound on the total number of LMI scalar variables for Theorem 1 is given by Table 1 and is equal to $\eta^{W C}=210$, moreover the true number in this example is just $\eta=67$.

\section{Conclusion}

This paper has provided an exact LMI condition for robust asymptotic stability of uncertain systems depending polynomially on a scalar parameter in both continuous-time and discrete-time cases. It has been shown that this condition requires a much smaller computational burden than existing exact LMI conditions.

\section{References}

[1] S. Boyd, L. El Ghaoui, E. Feron, and V. Balakrishnan. Linear Matrix Inequalities in System and Control Theory. SIAM, 1994. 
[2] A. T. Neto. Parameter dependent Lyapunov functions for a class of uncertain linear systems: an LMI approach. In IEEE Conference on Decision and Control, pages 2341-2346, Phoenix, Arizona, 1999.

[3] P.-A. Bliman. A convex approach to robust stability for linear systems with uncertain scalar parameters. SIAM Journal on Control and Optimization, 42(6):2016-2042, 2004.

[4] G. Chesi, A. Garulli, A. Tesi, and A. Vicino. Polynomially parameterdependent Lyapunov functions for robust stability of polytopic systems: an LMI approach. IEEE Transactions on Automatic Control, 50(3):365-370, 2005.

[5] C. W. Scherer. LMI relaxations in robust control. European Journal of Control, 12(1):3-29, 2006.

[6] R. C. L. F. Oliveira and P. L. D. Peres. Parameter-dependent LMIs in robust analysis: Characterization of homogeneous polynomially parameter-dependent solutions via LMI relaxations. IEEE Transactions on Automatic Control, 52(7):1334-1340, 2007.

[7] G. Chesi. Time-invariant uncertain systems: a necessary and sufficient condition for stability and instability via HPD-QLFs. Automatica, 46(2):471-474, 2010.

[8] G. Chesi, A. Garulli, A. Tesi, and A. Vicino. Homogeneous Polynomial Forms for Robustness Analysis of Uncertain Systems. Springer, 2009.

[9] X. Zhang, P. Tsiotras, and T. Iwasaki. Lyapunov-based exact stability analysis and synthesis for linear single-parameter dependent systems. International Journal of Control, 83(9):1823-1838, 2010.

[10] G. Chesi. LMI techniques for optimization over polynomials in control: a survey. IEEE Transactions on Automatic Control, 55(11):2500-2510, 2010 . 